Results The general pattern of results was similar in both studies. Pooling the studies, we found no increased risk of lung cancer for cleaning-related occupations (odds ratio and 95\% confidence interval (OR) for "ever employed in any cleaningrelated occupation": $1.0 ; 0.8-1.1)$ as compared to never cleaners. Among chemical exposures, we found a negative association of lung cancer with any occupational exposure to cleaning agents (OR: $0.9 ; 0.7-1.0)$, and biocides $(0.8 ; 0.7-1.0)$. In a secondary analysis, restricting to subjects with a history of asthma, the OR between lung cancer and long duration employment in a cleaning-related occupation was 2.1 (0.9 to 5.0). The OR of lung cancer and cleaning agents was also higher, yet non-significant, among asthmatics $(1.5 ; 0.8-2.8)$ than among non-asthmatics $(0.9 ; 0.7-1.1)$.

Conclusions People employed in cleaning-related occupations or who had worked with cleaning agents had no increased risk of lung cancer. There was a suggestion that this overall result masked a possible increased risk of lung cancer among asthmatics with cleaning-related exposures and/or occupations.

\section{OCCUPATIONAL EXPOSURE TO PAH AND LUNG CANCER RISK IN THE SYNERGY PROJECT}

${ }^{1} \mathrm{~N}$ G Guha, ${ }^{1}$ Olsson, ${ }^{2}$ Vermeulen, ${ }^{2}$ Kromhout, ${ }^{2}$ Almansa Ortiz, ${ }^{1}$ Vlaanderen, ${ }^{3}$ Pesch, ${ }^{3}$ Brüning, 'Schüz, 'Straif. 'IARC, Lyon, France; ${ }^{2}$ Institute for Risk Assessment Sciences (IRAS), Utrecht University, Utrecht, The Netherlands; ${ }^{3}$ IPA, Ruhr, Germany

\subsection{6/oemed-2013-101717.307}

Objectives To evaluate the association between occupational exposure to polycyclic aromatic hydrocarbons (PAH) and the risk of lung cancer, adjusted for tobacco smoking, in the SYNERGY project.

Methods The SYNERGY project pools data from 16 case-control studies conducted in Europe, Canada, China and New Zealand between 1985 and 2010. Lifetime occupational and smoking information was collected through interviews from 19,369 cases of lung cancer and 23,674 matched population or hospital controls. PAH exposure was estimated using a quantitative general population job-exposure matrix ('SYNJEM') based on five-digit ISCO-68 codes (4021 cases, 4077 controls ever exposed). Odds ratios (ORs) and 95\% confidence intervals $(95 \%$ $\mathrm{CI}$ ) of lung cancer risk were estimated using unconditional logistic regression models adjusted for age, sex, study centre, smoking behaviour, and ever employment in an occupation with known lung cancer risk.

Results We observed a modest increased risk of lung cancer associated with occupational exposure to $\mathrm{PAHs}$ according to various exposure metrics (ever/never, duration, cumulative dose, time since last exposure). The odds ratio (OR) for ever exposure to PAH was 1.09 (95\% CI, 1.04-1.15) overall, 1.08 (95\% CI, 1.02-1.15) among men and 1.20 (95\% CI, 1.051.38) among women. These results are further supported by significant exposure response-relationships ( $\mathrm{p}$-value for trend $<$ 0.05 for years of employment and cumulative exposure [(BaP) $\mu \mathrm{g} / \mathrm{m}^{3}$-years]). When stratified by histological subtype, increased risks and positive exposure response-relationships were apparent only for squamous cell carcinoma and small cell lung cancer.

Conclusions Our pooled analysis suggests that occupational exposure to PAH is associated with a modest increase in the risk of lung cancer, after adjustment for tobacco smoking and exposure to other occupational lung carcinogens.
308 LUNG CANCER AND EXPOSURES IN A REANALYSIS OF BRITISH COKE WORKERS

G Miller, Cherrie, Doust. Institute of Occupational Medicine, Edinburgh, United Kingdom

10.1136/oemed-2013-101717.308

Objectives Workers in coke ovens are potentially exposed to polycyclic aromatic hydrocarbons (PAHs), and studies have variously shown increased lung cancer risks. A study of two British company cohorts (NSF \& BSC), defined in 1967, reported in 1991 on a 20-year mortality follow-up of over 6,500 workers.

Analyses of mortality risks and exposures showed some statistically significant exposure-response relationships, as did analyses characterising exposure by time worked on coke oven tops. Limitations of the exposures were documented: notably, exposures to BSM were estimated only up to the start of follow-up (1967); and the exposures calculated made no allowance for cancer latency (time elapsing between exposure and increase in risk). The present reanalysis project was designed to bypass those limitations.

Methods Revised exposures to the general class of benzene-soluble materials (BSM) and to the specific marker benzo[a]pyrene $(\mathrm{B}[\mathrm{a}] \mathrm{P})$ were calculated from work histories up to 1976 , as were estimates of time worked on oven tops. Lung cancer mortality was analysed using Cox regression models, with time-dependent exposures lagged by 10 years to allow for latency.

Results Most results were not statistically significant. There were no significant trends with continuous measures of exposure to either BSM or B[a]P or of time worked in ovens job locations. With grouped exposures, at NSF, the highest category of $\mathrm{B}[\mathrm{a}] \mathrm{P}$ exposure had a statistically significant relative risk of 1.51 . In $\mathrm{BSC}$, the relative risk coefficient for working 5 or more years there was 1.81 , which was statistically significant (but lower than the equivalent value of 2.10 from the previous analyses).

Conclusions These results showed some signs consistent with an effect of coke ovens work on lung cancer risk, but there was little firm evidence of any real effect.

\section{LUNG CANCER AND EXPOSURE TO SILICA AND CEMENT DUSTS: THE ICARE}

${ }^{1}$ F G Guida, ${ }^{2}$ Sanchez, ${ }^{3}$ Menvielle, ${ }^{2}$ Lamkarkach, ${ }^{2}$ Cénée, ${ }^{3}$ Cyr, ${ }^{4}$ Févotte, ${ }^{3}$ Luce, ${ }^{2}$ Stücker. ${ }^{1}$ INSERM, Villejuif Cedex, France; ${ }^{2}$ INSERM U1018 Team 6, Villejuif, France; ${ }^{3}$ INSERM U1018 Team 11, Villejuif, France; ${ }^{4}$ UMRESTTE - Université Claude Bernard, Lyon, France

\subsection{6/oemed-2013-101717.309}

Objectives This work aims at assessing the risk of lung cancer associated with exposures to silica and cement dusts, which are among the most frequent occupational exposures for construction workers.

Methods ICARE is a large multicentre population-based casecontrol study conducted between 2001 and 2006 in 10 French départements with a general cancer registry. Complete occupational history was collected. The subjects' exposures to silica and cement dusts were determined using two Job-Exposure Matrices (JEM) specific for these substances. We used unconditional logistic regression adjusted for age, department, number of job periods, lifelong cigarette smoking, and asbestos exposure (assessed using a third JEM).

Results Analyses were conducted among men (1885 cases, 2368 controls) and concerned 18788 jobs. We found significant 